Thorax, 1983, 35, 686-689

\title{
Fungal endocarditis after homograft valve replacement: difficulties in diagnosis and treatment
}

\author{
ROBIN M RUDD, PAUL R HILL, PETER KOPELMAN, AND D JOHN PARKER \\ From the Departments of Cardiology and Cardiac Surgery, St George's Hospital, London
}

ABSTRACT Fungal endocarditis is an uncommon but important problem after cardiac surgery 6 Two cases of fungal endocarditis after homograft valve replacement are reported. In both patientsoo prolonged periods of antifungal chemotherapy with apparently satisfactory clinical responses? ultimately failed to eradicate the infection. Both patients remain free of infection two years after excision of the infected valves and further chemotherapy. The value of serial estimations of fungales antibody titres in diagnosis and treatment is demonstrated and the necessity for early operation iso emphasised.

Fungal endocarditis is an uncommon complication of open-heart surgery but it is important because of its grave prognosis. Since classical features of endocarditis may not be present and blood cultures may be persistently negative, the diagnosis is often not made until late in the course of the disease, sometimes after serious embolism has occurred. The following reports of two cases of fungal endocarditis after homograft aortic valve replacement illustrate these difficulties, and the use of fungal antibody titres and echocardiography as aids to diagnosis is discussed. In both patients antifungal chemotherapy alone failed to eradicate the infection and the need for treatment with a combination of early operation and antifungal agents is emphasised.

\section{Case reports}

CASE 1

A 53-year-old woman presented in 1967 with exertional dyspnoea. She had the signs of severe mitral stenosis and mild mixed aortic valve disease. A successful mitral valvotomy was performed and she remained well for eight years. In 1975 dyspnoea recurred and she was found to have developed severe aortic stenosis. In February 1976 her aortic valve was replaced with a fresh homograft, ${ }^{1}$ and a second open mitral valvotomy was performed. The immediate postoperative course was uneventful but during the

Address for reprint requests: Mr John Parker, St George's Hospital, Blackshaw Road, London SW17. next three months the patient was admitted on two occasions for investigation of pyrexia. No. cause was discovered: the blood count ando erythrocyte sedimentation rate remained normal and repeated blood cultures were sterile.

In July 1976 she was readmitted to hospita with a high fever and blood cultures at this time grew Candida parapsilosis. Candida antibodies $\overrightarrow{\vec{O}}$ were positive-the $C$ parapsilosis precipitin titre 3 was $1: 16$. An echocardiogram suggested mul-? tiple vegetations on the aortic homograft valve? (figure). Amphotericin B, to which the organism $\rightleftharpoons$ was fully sensitive, was administered by intra-음 venous infusion for 13 weeks. Adequate serum $x$ levels were recorded throughout this period and $\frac{\text { O }}{3}$ the total dose of amphotericin B was $4 \mathrm{~g}$. The pyrexia settled, the homograft remained competent without evidence of obstruction, and an echocardiogram showed clearance of the vegeta- $\frac{}{3}$ tions. Blood cultures were repeatedly sterile, but $D$ the $C$ parapsilosis precipitin titre remained positive at $1: 16$. The patient was asymptomatic and was discharged.

An embolus to the right leg resulted in readmission in February 1977. Thrombus removed at femoral embolectomy showed well-preserved and viable fungal hyphae. The $C$ parapsilosiso precipitin titre had risen to $1: 64$ but blood $\mathbb{\Phi}$ cultures were repeatedly sterile. The valve? remained competent clinically and the echocar- $\frac{T}{T}$ diogram was unchanged, showing no evidence $\frac{\vec{D}}{\mathbb{C}}$ of vegetations. At operation the aortic homo- $?$ graft was found to be functioning normally and $\varnothing$ the cusps were soft and pliable. However, on 


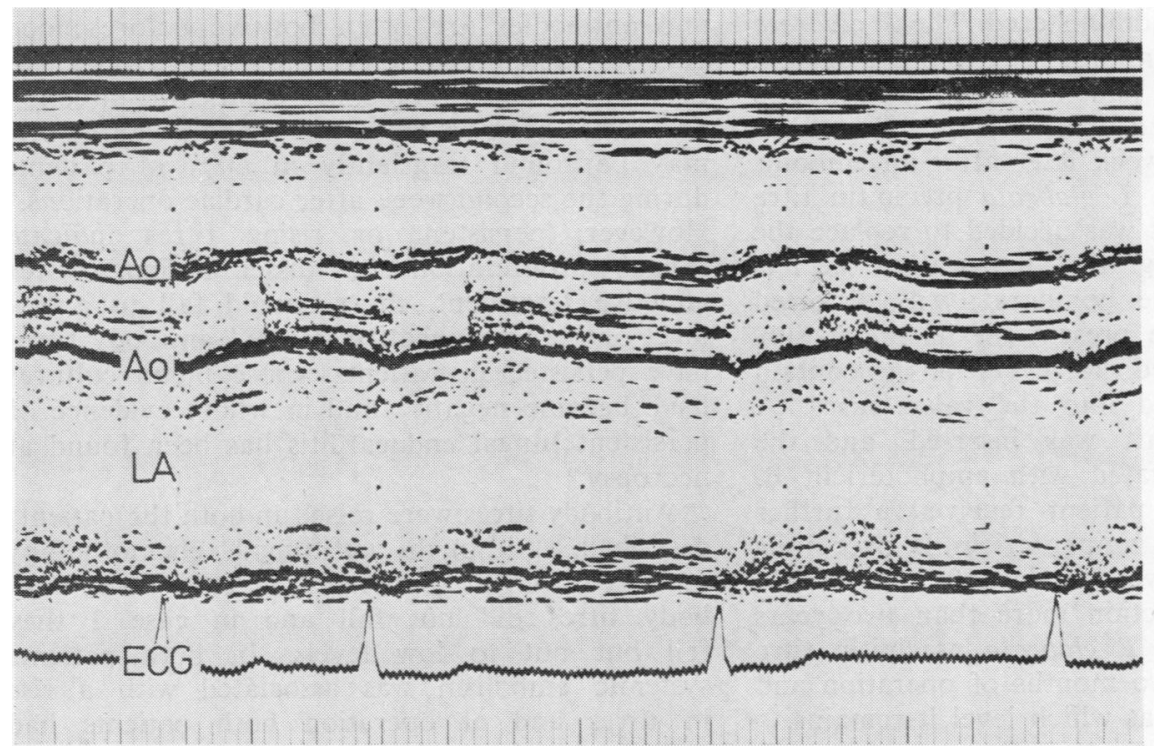

Figure Multiple echoes in the aortic root in diastole, suggestive of the presence of vegetations. $A o=$ aorta. $L A=$ left atrium, $E C G=$ electrocardiogram.

the undersurface of the right and non-coronary cusps some minute vegetations were found. Microscopy showed these to contain fungal hyphae which were eroding the ventricular surface of the cusps and extending deeply into the valve tissue. A further homograft replacement of the aortic valve was performed and the mitral valve was also replaced using a porcine heterograft (Hancock). The aortic root was irrigated with amphotericin $B$ in the course of the operation.

Recovery was satisfactory and the patient was discharged after receiving a further $2 \mathrm{~g}$ of amphotericin B over a period of seven weeks. She remains well over two years later. The $C$ parapsilosis precipitin titre fell to $1: 2$ within four weeks of the operation and subsequently to $1: 1$ at which level it remains more than two years after cessation of therapy.

\section{CASE 2}

A 26-year-old man was admitted to another hospital in April 1975 with a history of malaise and fever for one week. He had the signs of aortic regurgitation which had not been noted at previous medical examinations. A clinical diagnosis of infective endocarditis was made. Numerous blood cultures were sterile and despite treatment with intravenous benzyl penicillin and gentamicin the aortic regurgitation worsened. The development of left ventricular failure un- responsive to medical treatment necessitated early operation. A bicuspid aortic valve with a perforated right cusp was replaced with a fresh homograft. Histology of the valve showed inflammatory changes but no organisms were identified.

In June 1976 the patient was readmitted with a history of fever for two weeks. On examination Osler's nodes were noted. Blood cultures were negative and he was treated with benzyl penicillin. His temperature settled rapidly but after one week of treatment he suffered a cerebral embolus resulting in a right hemiparesis and dysphasia. Candida agglutinin antibodies were found to be positive at this stage but in view of the apparent response to penicillin this was not considered significant. Six weeks of treatment with benzyl penicillin were completed and an echocardiogram before discharge showed an apparently normal aortic valve.

In March 1977 there was a recurrence of the pyrexia and blood cultures grew Torulopsis glabrata, a fungus cross-reacting serologically with Candida species. Moderate aortic incompetence had developed and the echocardiogram suggested multiple vegetations on the homograft valve. The patient was treated with amphotericin $B$ by intravenous infusion, and oral flucytosine. Serum levels of each drug were kept at more than 10 times the minimum inhibitory concentration for the organism and a total of $1.5 \mathrm{~g}$ of 
amphotericin B was administered. The $T$ glabrata precipitin titre was initially $1: 64$, but after six weeks of treatment had fallen to $1: 16$. The patient was discharged on flucytosine alone, but was readmitted one week later after an embolus to his right leg. The $T$ glabrata precipitin titre had risen to $1: 32$. It was decided to replace the diseased aortic homograft. At operation the left coronary cusp of the homograft was distorted and vegetations were present on its under surface. Histology showed the vegetations to contain fungi which extended into the valve cusps. A new aortic homograft was inserted, and the aortic root was irrigated with amphotericin B. After operation the patient received a further $0.8 \mathrm{~g}$ of intravenous amphotericin $\mathrm{B}$ over four weeks and oral flucytosine for 10 months. He remains free of infection more than two years after operation. The $T$ glabrata precipitin titre fell to $1: 8$ within two months of operation and subsequently to $1: 1$ at which level it remains.

\section{Discussion}

Fungaemia can occur after open-heart surgery or during the course of prolonged intravenous therapy. ${ }^{2}$ The route of entry of the organism is uncertain. Candida species and Torulopsis glabrata are found as commensals in the human gastrointestinal tract. Invasiveness is enhanced when the bacterial flora is disturbed by broad spectrum antibiotics, and these are given prophylactically at the time of the operation and for five days afterwards. It is thought unlikely that the homograft valves were infected by fungi before insertion, as the method of preparation involves storage in a solution containing a high concentration of nystatin as well as antibacterial agents. In addition, pieces of valve tissue are cultured for bacteria and fungi before the valve is accepted for surgical use. ${ }^{1}$

It is unwise to ignore the isolation of fungi from the blood as fungal endocarditis has been recorded up to 18 months after apparently transient fungaemia. ${ }^{3}$ Classical signs of endocarditis often do not occur until late in the course of the disease, and in a significant proportion of cases may be absent. Arterial emboli may be the first manifestation and they occur more commonly and tend to be larger than in bacterial endocarditis. ${ }^{45}$

The prognosis of fungal endocarditis is poor and a means of earlier diagnosis is needed. Echocardiography can reliably identify vegetations but it is an insensitive test. ${ }^{67}$ Serological tests may give an early indication of fungal infection, and antibodies are often positive before blood음 cultures. The detection of antibodies does not $\frac{\bar{\sigma}}{\bar{\omega}}$ necessarily imply clinically significant infection $\vec{\sigma}_{\bar{\sigma}}$ and it has been reported that Candida antibodies $\varrho$ may be found transiently in $40 \%$ of patients during the second week after cardiac operations. ${ }^{8} \overrightarrow{ }$ However, persistent or rising titres indicate fungal proliferation or dissemination. After $\vec{\omega}$ effective treatment, titres should fall to a low? level or become negative. Raised antibody titres $\vec{x}$ have persisted in patients whose blood cultures $\mathrm{c}$ have become negative and in whom evidence of 6 persistent fungal endocarditis has been found at necropsy. ${ }^{9}$

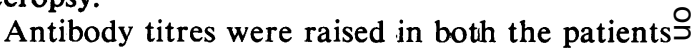
described. Although antifungal chemotherapy $\vec{c}$ produced clinical remissions, in case 1 the anti- $\mathbb{\Phi}$ body titres did not fall and in case 2 they fell but not to low levels. In both patients systemic embolism was associated with a riseo in titres and at operation both patients had $\overrightarrow{{ }_{0}}$ viable fungi in their homografts. Afteroo operation the titres fell to and remained at low levels. Precipitin titres wereo assayed by counter-immunoelectrophoresis using a preparation containing cytoplasmic antigens. $\bar{O}$ Agglutinin titres were obtained using whole cells. Precipitin and agglutinin titres moved in theo same direction but the precipitin titre was more $\overrightarrow{\vec{A}}$ stable, and changes were consistently associated 3 with changes in the clinical condition.

In reported medical cures of Candida endo carditis the diagnosis has usually been based on the finding of fungaemia alone, without definite evidence of fungal invasion of heart valves. ${ }^{10} \times$ Several reported cures have been followed by relapse up to one year after treatment was dis continued. ${ }^{11}$ Failure of chemotherapy may beo the result of poor penetration of amphotericin $\mathrm{B}^{3}$ into the vegetations of fungal endocarditis. $\mathrm{Cul}$ 윽 tures positive for Candida have been obtained $\triangle$ after incubation of homogenised vegetation in medium containing $12.8 \mu \mathrm{g} / \mathrm{ml}$ of amphotericin B for 168 hours. ${ }^{12}$

The best chance of cure and of preventing potentially dangerous arterial embolism is pro-w vided by excision of the infected valve and removal of any other lesions. The infected areao should be irrigated with amphotericin B at theo time of operation, and this should be followed? by systemic antifungal therapy. ${ }^{13}$ Antibody titreso should be estimated regularly until at least a yeap after cessation of chemotherapy: if treatmen has been successful titres should fall to a low level. A significant rise in titres or persistence of high titres should be regarded as indicating 
the need for further treatment.

We thank Professor DWR Mackenzie, PhD, Director of the Mycological Reference Laboratory, London School of Hygiene and Tropical Medicine, for his help with the mycology.

\section{References}

1 Ross DN, Martelli V, Wain WH. Allograft and autograft valves used for aortic valve replacement. In: Ionescu MI (ed). Tissue heart valves. London: Butterworths, 1979; 127-72.

2 Ellis CA, Spivack ML. The significance of Candidemia. Ann Intern Med 1967; 67:511-22.

3 Rubinstein E, Noriega E, Simberkoff MS, Holzman R, Rahal JJ Jr. Fungal endocarditis: analysis of 24 cases and review of the literature. Medicine (Baltimore) 1975; 54:331-44.

4 Montague NT, Sugg WL. Candida endocarditis with femoral emboli: treatment with surgery and 5-fluorocytosine. J Thorac Cardiovasc Surg 1974; 67:322-7.

5 Utley JR, Mills J, Roe BB. The role of valve replacement in the treatment of fungal endocarditis. J Thorac Cardiovasc Surg 1975; 69: 255-8.
6 Dillon JC, Feigenbaum H, Konecke LL, Davis RH, Chang S. Echocardiographic manifestations of valvular vegetations. Am Heart J 1973; 86: 698-704.

7 Wann LS, Dillon JC, Weyman AE, Feigenbaum R. Echocardiography in bacterial endocarditis. $N$ Engl J Med 1976; 295:135-9.

8 Murray IG, Buckley HR, Turner GC. Serological evidence of Candida infection after open heart surgery. J Med Microbiol 1969; 2:463-8.

9 Seelig MS, Speth CP, Kozinn PJ, Taschdjian CL, Toni EF, Goldberg P. Patterns of Candida endocarditis following cardiac surgery: importance of early diagnosis and therapy (an analysis of 91 cases). Progr Cardiovasc Dis 1974; 17:125-60.

10 Mayrer ER, Brown A, Weintraub RA, Ragni M, Postic B. Successful medical therapy for endocarditis due to Candida parapsilosis. Chest 1978; 73:546-9.

11 Galgiani JN, Stevens DA. Fungal endocarditis: need for guidelines in evaluating therapy. J Thorac Cardiovasc Surg 1977; 73:293-6.

12 Rubinstein E, Noriega ER, Simberkoff MS, Rahal JJ Jr. Tissue penetration of amphotericin B in Candida endocarditis. Chest 1974; 66:376-7.

13 Kay JH, Bernstein S, Tsuji HK, Redington JV, Milgram M, Brem T. Surgical treatment of Candida endocarditis. JAMA 1968; 203:621-6. 